\title{
AFLATOXINAS EN ALIMENTOS Y EXPOSICIÓN DIETARIA COMO FACTOR DE RIESGO PARA EL CARCINOMA HEPATOCELULAR
}

\author{
Erika Melissa Londoño-Cifuentes ${ }^{1}$ \\ María Marcela Martínez-Miranda²
}

\section{RESUMEN}

Introducción: El consumo de cereales, frutos secos, especias y leche de vaca es elevado a nivel global. Estos alimentos son susceptibles a la contaminación biológica, incluyendo la causada por hongos del género Aspergillus, que producen metabolitos tóxicos llamados aflatoxinas, los cuales ocasionan efectos adversos en la salud. Las aflatoxinas B1 y M1 han sido clasificadas por la Agencia Internacional de Investigación del Cáncer (IARC) en el Grupo I como carcinógenos para humanos. El presente artículo muestra la ocurrencia de aflatoxinas en algunos alimentos de consumo humano y su relación con el desarrollo de carcinoma hepatocelular.

Métodos: Se llevó a cabo una búsqueda bibliográfica en las bases de datos: Science Direct, Scopus, Pubmed y Web of Science. Se utilizaron palabras como "risk assessment and aflatoxin", "ocurrence and aflatoxins", "aflatoxins and hepatocellular carcinoma", "aflatoxins and foodstuffs".
Resultados: Con base en los estudios consultados se demuestra que la ocurrencia de aflatoxinas es alta, principalmente en cereales como arroz, maíz y trigo. Además, las investigaciones relacionadas con la evaluación de la exposición destacan el vínculo existente entre el consumo frecuente de estas micotoxinas y el riesgo de desarrollar carcinoma hepatocelular, especialmente en personas que han contraído el virus de la hepatitis B.

Conclusión: La presencia de aflatoxinas en alimentos ha generado alarmas de seguridad alimentaria a nivel mundial. Aunque se han creado algunas regulaciones, en países donde hay mayor exposición a estas micotoxinas, existe poco o ningún control de calidad. Por ello, es importante generar mecanismos de vigilancia pertinentes para disminuir el riesgo de contaminación fúngica y alimentos con aflatoxinas.

Palabras clave: aflatoxinas, prevalencia, medición de riesgo, carcinoma hepatocelular.

\footnotetext{
${ }^{1}$ Joven Investigadora Colciencias, Ingeniera de Alimentos. Universidad de Caldas. Correo electrónico: melissa182s@ gmail.con

${ }_{2}$ Docente Asistente. Universidad de Caldas. Magíster en Microbiología. Grupo de Investigación en Cromatografía y Técnicas Afines (GICTA). Correo electrónico: marcela.martinez@ucaldas.edu.co
} 


\section{AFLATOXINS IN FOOD AND DIETARY EXPOSURE AS A RISK FACTOR FOR HEPATOCELLULAR CARCINOMA}

\section{ABSTRACT}

Introduction: Consumption of grains, nuts dried fruits, spices and cow's milk is high globally elevated. These foods are susceptible sensitive to biological contamination, including that caused by fungi of the genus Aspergillus, which produce secondary toxic metabolites called aflatoxins, which cause adverse health effects. The aflatoxin B1 and M1 have been classified by the International Agency for Research on Cancer (IARC) in Group I as carcinogenic to humans. This article shows the occurrence of aflatoxins in some foods for human consumption and its relationship to the development of hepatocellular carcinoma. Methods: A bibliographic search was carried out in the databases: Science Direct, Scopus, Pubmed and Web of Science. Words like "risk assessment and aflatoxin",

\section{INTRODUCCIÓN}

De acuerdo con la Food Agricultural Organization of the United Nations (FAO), la principal fuente de energía obtenida por la ingesta de alimentos mundial son los cereales como el arroz, maíz, trigo, sorgo y cebada; estos presentan mayor disponibilidad para su consumo en países exportadores y, aunque su producción proyectada a futuro se ve disminuida, siguen siendo productos básicos para la alimentación humana (1). Por otra parte, si bien el consumo de frutos secos, especias y leche de vaca globalmente es menor, hay regiones donde culturalmente se reporta alta ingesta de estos alimentos. Se ha encontrado que estos productos se ven afectados por distintos tipos de contaminación biológica, dentro de las cuales se presenta la formación de metabolitos tóxicos llamados micotoxinas "occurrence and aflatoxins", "aflatoxins and hepatocellular carcinoma", "aflatoxins and foodstuffs" were used. Results: Based on the studies conducted it was demonstrated that the occurrence of aflatoxins is high, especially in cereals grains such as rice, corn, and wheat. In addition, research related to exposure assessment highlighted the link between frequent consumption of these mycotoxins and the risk of developing hepatocellular carcinoma, especially in people who have contracted hepatitis B virus. Conclusion: The high prevalence of aflatoxins in food has generated food security safety alarms worldwide. Although some regulations have been created, in countries where there is greater more exposure to these mycotoxins there is little or no quality control. It is Therefore, it is important to create relevant monitoring mechanisms so that the to reduce the risk of fungal contamination and food with aflatoxins. possible cases of liver cancer may be lower.

Key words: aflatoxins, prevalence, risk assessment, carcinoma, hepatocellular.

a partir de diferentes especies de hongos, que crecen bajo condiciones ambientales apropiadas durante su cosecha o almacenamiento (2).

Las micotoxinas son metabolitos tóxicos secundarios producidos por ciertas especies de hongos filamentosos (especialmente del género Aspergillus) que cuando son ingeridas, inhaladas o absorbidas a través de la piel pueden ocasionar enfermedades e, incluso, la muerte en seres humanos y animales (3). Las aflatoxinas se encuentran dentro de las principales micotoxinas, existiendo aproximadamente 20 tipos diferentes, sin embargo, las aflatoxinas B1 (AFB1), B2 (AFB2), G1 y G2 son las que se encuentran comúnmente en alimentos, principalmente en granos y frutos secos (4); mientras que las aflatoxinas M1 (AFM1) y M2 son derivados metabólicos de las aflatoxinas 
B1 y B2 respectivamente, que se excretan en la orina y leche de quienes han consumido piensos contaminados (5). La AFM1 excretada en la leche de vaca tiene propiedades tóxicas similares a las de la AFB1 (6), además se ha documentado que AFB1 y AFM1 son más tóxicas y carcinógenas que las otras, ya que han sido clasificadas por la Agencia Internacional de Investigación del Cáncer (IARC) en el grupo I como carcinógenos para humanos (7).

Teniendo en cuenta que el consumo de cereales, frutos secos, especias y leche de vaca son elevados en el mundo, es importante conocer los estudios que se han realizado en materia de ocurrencia de aflatoxinas en estos alimentos, la ingesta de los mismos y los posibles efectos de la exposición a estas micotoxinas en la salud humana; pues se ha comprobado que mediante mecanismos oxidativos en el organismo, después de ser ingeridas, las aflatoxinas pueden activarse en el hígado y convertirse en aductos que se unen al ADN y tienen un efecto carcinogénico y hepatotóxico en las células somáticas, acumulándose durante años según la frecuencia de consumo de alimentos contaminados (8). Teniendo en cuenta lo anterior, el propósito de este artículo es presentar una revisión sobre la prevalencia de las aflatoxinas en diversos alimentos en el mundo, la evaluación de la exposición y su relación con el desarrollo del carcinoma hepatocelular.

\section{Prevalencia de aflatoxinas en alimentos Aflatoxinas en arroz}

El arroz es uno de los cereales más cultivados y consumidos mundialmente llegando a un volumen cercano a 740 millones de toneladas producidas en 2015 (9).

En 2001 se estudió la incidencia de aflatoxinas en productos colombianos seleccionados, incluido el arroz, y se demostró la presencia de aflatoxinas totales en el $10 \%$ de las muestras analizadas, con un valor promedio de $7,1 \mu \mathrm{g} / \mathrm{kg}$. Se concluyó que a pesar de que los niveles hallados estuvieron dentro de los límites máximos establecidos en la mayoría de países $(20 \mu \mathrm{g} / \mathrm{kg})$, existe el riesgo de desarrollar enfermedades (10).

En 2007, Nguyen y colaboradores investigaron la ocurrencia de AFB1 y otras micotoxinas en arroz de cinco provincias de la región central de Vietnam y encontraron que la AFB1 estaba presente en el $51 \%$ de las muestras analizadas con un promedio de $3,3 \mu \mathrm{g} / \mathrm{kg}$ y que el nivel de contaminación estaba afectado por la estación del año, particularmente la época lluviosa (11).

En otros estudios se encontraron niveles promedio de 0,45 $\mu \mathrm{g} / \mathrm{kg}$ para AFB1 y 9,86 $\mu \mathrm{g} /$ kg para AFB2 en arroz comercializado en Austria (12); niveles promedio de 0,60 y $0,65 \mu \mathrm{g} / \mathrm{kg}$ de AFB1 y aflatoxinas totales, respectivamente, en seis provincias de China (13) y $8,23 \mu \mathrm{g} / \mathrm{kg}$ para AFB1 y 19,54 $\mu \mathrm{g} / \mathrm{kg}$ para aflatoxinas totales en Pakistán (6). Los resultados obtenidos en estos estudios varían de acuerdo con las zonas climáticas y el manejo durante la cosecha y poscosecha. Sin embargo, la mayoría de las concentraciones halladas no sobrepasan los límites establecidos por la Comisión Europea en la Regulación 1881/2006 para arroz de consumo directo, que son de $2 \mu \mathrm{g} / \mathrm{kg}$ para AFB1 y $4 \mu \mathrm{g} /$ $\mathrm{kg}$ para aflatoxinas totales (14).

Si bien, en su mayoría existe contaminación a bajas concentraciones y se asume que los productos son generalmente seguros, la prevención es fundamental para controlar la ocurrencia de estas micotoxinas, pues a pesar de que las muestras que superan los límites permitidos son pocas, hay evidencia del riesgo potencial existente para la salud humana.

Lutfullah y Hussain (15) estudiaron los niveles de aflatoxinas en algunos cereales de Pakistán y encontraron un porcentaje de contaminación del $25 \%$ en las muestras de arroz analizadas, de las cuales dos tenían niveles de aflatoxinas por encima de los límites establecidos en la regulación de la Unión Europea para aflatoxinas totales $(4 \mu \mathrm{g} / \mathrm{kg})(15)$. 
Gracias a la clasificación de AFB1 como carcinógeno humano (16), diversas investigaciones se enfocan en determinar la exposición a esta micotoxina a través de alimentos de alto consumo demostrando que, después del maíz, el arroz es el principal responsable de la ingesta de AFB1 en países como Corea (17). No obstante, en Japón los principales contribuyentes de la ingesta de AFB1 también son el maní y derivados, la pimienta roja y blanca, las almendras y el trigo (18).

Es importante destacar que, aunque el arroz es consumido principalmente cocido, las aflatoxinas tienen una composición química que les provee gran estabilidad al calor, por lo cual podrían mantenerse en la mayoría de alimentos listos para el consumo. Sin embargo, se ha reportado que la clasificación, limpieza, molturación y tratamientos térmicos como el horneado, la cocción, el tostado y la extrusión, entre otros, podrían reducir el contenido de estas micotoxinas en porcentajes significativos (superiores al 70\%) (19). Por ejemplo, para el caso de AFB1 se disminuiría a concentraciones menores a $20 \mu \mathrm{g} / \mathrm{kg}$ (20). Con estos estudios podría decirse que procesar el arroz para consumirlo puede reducir el riesgo de contaminación por aflatoxinas, sin embargo, es indispensable realizar más análisis y controles que garanticen estos resultados.

\section{Aflatoxinas en maíz}

Según la FAO, el maíz está clasificado como el segundo cultivo de mayor producción mundial, después del trigo, con una producción estimada hasta el 2012 de 872,8 millones de toneladas en el mundo (21).

El maíz y sus derivados son muy propensos a la contaminación por hongos (Fusarium y Aspergillus) productores de micotoxinas, especialmente aflatoxinas (22). En Panamá se encontró, en la mayoría de los casos, una contaminación mayor al $50 \%$ de las muestras analizadas para maíz sin procesar. En una de las variedades se encontró que el $27,3 \%$ de las colonias aisladas eran toxigénicas y contenía $1290 \mu \mathrm{g} / \mathrm{kg}$ de AFB1, excediendo los límites establecidos para que el alimento fuese inocuo toxicológicamente según la OMS (5 $\mu \mathrm{g} / \mathrm{kg}$ ) (23).

En México se realizó una inspección de aflatoxinas en maíz cultivado, almacenado y transportado en uno de sus estados, donde si bien algunas de las concentraciones halladas no excedían los límites establecidos en su legislación, todas las muestras presentaron contaminación. Además, del maíz recolectado directamente de los cultivos, se encontró que el $72 \%$ de éste presentaba concentraciones que superaban los niveles establecidos por la Norma Oficial Mexicana NOM-247-SSA1-2008 de 20 $\mu \mathrm{g} / \mathrm{kg}$ para aflatoxinas totales en cereales para consumo humano (24), estando en niveles entre $20-233 \mu \mathrm{g} / \mathrm{kg}(25)$.

Los cereales para desayuno (que contienen maíz, arroz y trigo) están frecuentemente contaminados por aflatoxinas (26). De acuerdo con un estudio realizado en Atenas, donde analizaron muestras comerciales de estos, se hallaron niveles de estas micotoxinas entre 0,05 y $4,30 \mu \mathrm{g} / \mathrm{kg}$ (27), mientras que en Ghana estuvieron entre 0,18 y $36,1 \mu \mathrm{g} / \mathrm{kg}$ (28), siendo más altos en cereales a base de maíz. Es importante destacar que este tipo de productos son mayormente consumidos por población infantil y los niveles sobrepasan el límite permitido de AFB1 $(0,1 \mu \mathrm{g} / \mathrm{kg})$ según la Comisión Europea (29) en esas investigaciones. Aunque esto depende de la localización y manejo que se le dé al producto, pues se han encontrado en España valores mínimos entre 0,08-0,13 $\mu \mathrm{g} / \mathrm{kg}(30)$.

Diferentes estudios acerca de la contaminación por aflatoxinas en maíz y derivados concuerdan con el hallazgo de niveles de concentración superiores a los permitidos según la UE $(5 \mu \mathrm{g} /$ $\mathrm{kg}$ )(14). Majeed et al. (6) encontraron en Pakistán niveles de AFB1 entre 4,8-9,8 $\mu \mathrm{g} / \mathrm{kg}$, y el porcentaje de muestras que excedían los límites permitidos fue del 16\%; mientras que en tres áreas de China el valor medio de concentración de AFB1 fue 13,5 $\mu \mathrm{g} / \mathrm{kg}$ (31). 
Debido a la recurrencia de casos de aflatoxicosis en Kenia, Kilonzo (32) estudiaron los niveles de aflatoxinas en maíz y derivados, encontrando altas concentraciones de éstas en granos de maíz (entre 18-480 $\mu \mathrm{g} / \mathrm{kg}$ ) y en harinas de maíz (6-30 $\mu \mathrm{g} / \mathrm{kg})$. Estos casos reflejan la ausencia de regulaciones estrictas para micotoxinas, especialmente donde el contenido de aflatoxinas fue demasiado alto.

\section{Aflatoxina M1 en leche de vaca}

Diversos países han hecho estudios acerca de los niveles de AFM1 en muestras de leche de vaca y derivados como leche en polvo, quesos, helados, entre otros, con el fin de exponer el riesgo existente por el consumo de estos alimentos y establecer si se realizan los procesos de vigilancia y control adecuadamente, pues se ha demostrado que ni siquiera los tratamientos térmicos utilizados en la industria (como la pasteurización y esterilización), son capaces de inactivar esta micotoxina (33).

En México se reportó presencia de AFM1 en muestras de leche cruda (9 muestras), UHT (20 muestras) y orgánica (15 muestras), por encima del límite máximo permitido de 0,05 $\mu \mathrm{g} / \mathrm{L}$ en la Unión Europea. Las medianas de AFM1 encontradas en muestras de leche cruda, UHT y orgánica fueron 16,2; 16,1 y 23.,1 $\mu \mathrm{g} / \mathrm{kg}$, respectivamente. El porcentaje de muestras por encima del límite máximo fue menor en leche de producción orgánica $(20 \%)$ comparada con las leches crudas y UHT que estuvieron por encima del 50 y $60 \%$, respectivamente (34).

Hernández y colaboradores realizaron un estudio para cuantificar AFM1 en 48 muestras de leche entera provenientes del Caribe colombiano y AFB1 en pienso suministrado a las vacas. Los resultados mostraron que ninguna de las muestras analizadas sobrepasó el límite permisible para esta micotoxina de acuerdo con la legislación implementada en Colombia $(0,5$ $\mu \mathrm{g} / \mathrm{L})$, pero al analizar los resultados desde el límite establecido por la Unión Europea $(0,05$ $\mu \mathrm{g} / \mathrm{L})$ se encontró que el 8,33\% de las muestras estuvo por encima del nivel máximo permitido. Se demostró que existió una influencia del clima de acuerdo con las cantidades de AFM1 encontradas, en donde las muestras de verano presentaron un nivel de contaminación mayor que las muestras de invierno. En las muestras de pienso analizadas se hallaron cantidades de AFB1 en concentraciones que no superaron el nivel regulatorio (35).

En Argentina se evaluó la concentración de AFM1 en muestras de leche de vaca y se estimó que el promedio de ésta se ubicó en el nivel máximo permitido por la normativa MERCOSUR $(0,5 \mu \mathrm{g} / \mathrm{kg})$ pero fue mucho más alto que el establecido por las regulaciones de la Unión Europea $(0,05 \mu \mathrm{g} / \mathrm{kg})(14)$. Concluyeron que entre el $0,66 \%$ y el $5,06 \%$ de la leche analizada superó el nivel máximo permitido por MERCOSUR para AFM1, y entre el 32,65\% y $66,84 \%$ estarían excedidos según la legislación europea (36).

En Paraná (Brasil) se determinó la presencia y concentración de AFM1 en leche comercial UHT, se encontró que el $87,5 \%$ de las muestras estaban contaminadas con AFM1 y el 2,6\% superaban los límites máximos establecidos por la Unión Europea; concluyeron que la concentración de esta toxina fue diferente según las diferentes estaciones, mostrando valores más altos durante el otoño (37).

Por otro lado, en Kosovo se halló baja incidencia de AFM1 en muestras de leche cruda y UHT (0,0052-0,0266 $\mu \mathrm{g} / \mathrm{L})$. Sin embargo, se concluyó que se hace esencial continuar con los programas de vigilancia de AFM1 en leche debido a la variabilidad climática y a que las zonas de mayor humedad y producción de maíz y trigo, usados para producción de piensos, fue donde hubo mayor prevalencia de AFM1 (38).

En Croacia, el 98,4\% de las muestras de leche contenían AFM1 en niveles inferiores a los permitidos en la Unión Europea (39). En Grecia, un estudio mostró que los niveles de esta 
micotoxina en leche estuvieron por debajo de $0,0182 \mu \mathrm{g} / \mathrm{L}(40)$, con lo cual se expone que en ambos países las tasas de contaminación son bajas.

Una investigación mostró en leche cruda y queso fresco de Libia, al norte de África, altos niveles de AFM1 en un rango entre 0,030-3,13 $\mu \mathrm{g} / \mathrm{L}$; revelando que las diferencias entre países están dadas por diversas variables como clima, suelo, control de plagas, control poscosecha y legislación vigente que establezca e inspeccione estos casos (41).

En Costa Rica, Chavarría y colaboradores documentaron en 2015 que los niveles de AFM1 hallados en leche de vaca se encontraban entre 0,019-0,629 $\mu \mathrm{g} / \mathrm{L}$, demostrando así inadecuados controles toxicológicos de calidad en la industria lechera de ese país (42).

Por otra parte, en Argelia, debido a la poca información sobre la contaminación de leche por AFM1, Redouane-Salah et al. (43) investigaron su presencia en muestras de leche cruda, leche en polvo y leche reconstituida. Detectaron que el $11 \%$ del total de las muestras estaban contaminadas a niveles de $0,001 \mu \mathrm{g} / \mathrm{L}$ y entre $0,009-0,103 \mu \mathrm{g} / \mathrm{L}$ para leche cruda y en polvo, respectivamente. Sin embargo, la mayor ocurrencia de AFM1 se encontró en la leche en polvo importada de Argentina (29\%). Así se concluyó que, aunque fue sólo una la muestra de esta última la que excedió los límites establecidos por la Unión Europea $(0,05 \mu \mathrm{g} / \mathrm{L})$, su presencia justifica una vigilancia continua.

\section{Aflatoxinas en especias y frutos secos}

Las especias son mundialmente utilizadas para condimentar y preparar alimentos de mesa, algunas de ellas pueden contener aceites esenciales con actividad antimicrobiana, sin embargo muchas otras son susceptibles de contaminación por hongos filamentosos y, seguidamente, por micotoxinas (3).

La paprika es una especia que proviene del pimentón rojo, obtenida por la molienda de las vainas de los frutos secos (44). Los estudios en ésta se han realizado continuamente pues es una especia de gran consumo. Hernández Hierro y García-Villanova (45) encontraron niveles de AFB1 entre 0,7-3,8 $\mu \mathrm{g} / \mathrm{Kg}$ para este producto en España. En Brasil, Shundo et al. (44) hallaron rangos de contaminación entre $0,5-7,3 \mu \mathrm{g} / \mathrm{Kg}$.

Cho et al. (46), en Corea, hallaron niveles bajos de aflatoxinas para curry $(0,13-0,46 \mu \mathrm{g} / \mathrm{kg})$ y pimienta roja $(0,08-4,45 \mu \mathrm{g} / \mathrm{kg})$. Sin embargo, en Turquía la contaminación en pimienta negra y roja es mucho mayor pues, según la investigación realizada por Colak (47) se presentaron rangos entre 9,8-10,3 $\mu \mathrm{g} / \mathrm{kg}$ y 1,9$35,5 \mu \mathrm{g} / \mathrm{kg}$, respectivamente.

Zhao y Schaffner (3) incluyeron en su estudio pimienta, chile, canela, anís, hinojo, curry, comino y jengibre; y encontraron que las especias con mayor concentración de AFB1 fueron el chile $(27,52 \mu \mathrm{g} / \mathrm{kg})$ y la pimienta $(4,51$ $\mu \mathrm{g} / \mathrm{kg})$, donde el primero sobrepasó el límite de regulación europea que es de $5 \mu \mathrm{g} / \mathrm{kg}$ (14).

Aunque el consumo de especias varía de un continente a otro, es importante garantizar en cada país que este tipo de productos (importados o no) estén libres de contaminación y cumplan las legislaciones para disminuir el riesgo en la salud.

Por otra parte, gracias a las características nutricionales y la larga vida útil que poseen los frutos secos, estos son consumidos como una buena alternativa de alimentación, especialmente durante temporadas de invierno (48); sin embargo, se ha demostrado que este tipo de productos son los más susceptibles a contaminación fúngica $\mathrm{y}$, consecuentemente, a la producción de aflatoxinas (49).

En Malasia se reportó la incidencia de aflatoxinas en maní y sus subproductos y se descubrió contaminación en niveles desde 16,6 $\mu \mathrm{g} / \mathrm{kg}$ hasta $711 \mu \mathrm{g} / \mathrm{kg}$. Al ser tan altos, se considera la hipótesis de que su ocurrencia se debe a que 
en este país se presentan elevadas temperaturas y humedad, lluvias durante la cosecha e inundaciones, condiciones que pueden conllevar a la proliferación de hongos toxigénicos y posterior producción de aflatoxinas (50). De la misma manera, en Corea se halló un rango de concentración entre $0,2-28,2 \mu \mathrm{g} / \mathrm{kg}$ en los mismos productos (51).

Neamatallah y Serdar investigaron la incidencia de contaminación por aflatoxinas en algunos frutos secos en la ciudad santa de La Meca (52). La media hallada para maní $(16,5 \mu \mathrm{g} /$ $\mathrm{kg})$, pistachos $(16,6 \mu \mathrm{g} / \mathrm{kg})$, nueces $(3,4 \mu \mathrm{g} /$ $\mathrm{kg})$, marañón $(1,6 \mu \mathrm{g} / \mathrm{kg})$, avellanas $(3,5 \mu \mathrm{g} /$ $\mathrm{kg})$ y almendras $(3,5 \mu \mathrm{g} / \mathrm{kg})$ demostró que únicamente el maní y los pistachos sobrepasaron el límite establecido por la Comisión de la Unión Europea para aflatoxinas totales $(4 \mu \mathrm{g} / \mathrm{kg})(53)$.

En países como Pakistán, el consumo de frutos secos se incrementa durante el invierno. Con el fin de conocer los niveles de contaminación en ellos, Masood et al. (54) analizaron muestras de maní, nueces, pistachos y semillas de frutas como sandía y melón y encontraron que el 75\% de éstas contenían entre $8-10 \mu \mathrm{g} / \mathrm{kg}$. Por otra parte, la exposición a AFB1 a través de frutos secos fue evaluada para la población belga en 2015 , las concentraciones encontradas fueron de $8 \mu \mathrm{g} / \mathrm{kg}$ para almendras y pistachos, $5 \mu \mathrm{g} / \mathrm{kg}$ para avellanas y $2 \mu \mathrm{g} / \mathrm{kg}$ para maní e higos (55). Asimismo, Mupunga et al. (56) determinaron el grado de contaminación por aflatoxinas totales en maní y mantequilla de maní distribuida y consumida en Zimbabue, hallaron un rango entre 6,1 y $247 \mu \mathrm{g} / \mathrm{kg}$; AFB1, que fue la más prevalente al tener concentraciones entre 3,7 y $191 \mu \mathrm{g} / \mathrm{kg}$.

\section{Aflatoxinas y carcinoma hepatocelular}

A partir de la década de 1970 se inició la recopilación de pruebas acerca de la relación entre la exposición a aflatoxinas y la incidencia de cáncer en células hepáticas. En 1994, en una provincia de China se llevó a cabo un estudio de seguimiento de marcadores urinarios para evaluar la exposición a aflatoxinas y el riesgo de padecer CHC (57). Con el fin de conocer la relación existente entre estos, se analizaron un total de 18.244 muestras de orina de personas entre 45 y 64 años de edad, en los que la incidencia de padecer esta enfermedad era mayor en aquella época. En las muestras fueron encontradas altas concentraciones de AFM1 y AFP1 (metabolitos urinarios derivados de la AFB1 (58)). Las asociaciones halladas entre la presencia de estas micotoxinas en orina y el antígeno del VHB fueron especialmente elevados para los individuos que obtuvieron resultados positivos en ambos biomarcadores; sin embargo, un análisis realizado en los casos de personas con CHC no reveló ninguna asociación estadísticamente significativa entre el riesgo de padecer esta enfermedad y la ingesta de alimentos contaminados con aflatoxinas, teniendo en cuenta el tipo de alimento y la frecuencia de consumo de estos. No obstante, se resaltó la importancia de indagar más en este campo y usar mediciones de biomarcadores en estudios epidemiológicos.

Según Ferlay et al., en el 2012 el carcinoma hepatocelular ( $\mathrm{CHC}$ ) era la segunda causa de muerte por cáncer en el mundo, responsable de cerca de 745.000 muertes en ese año (9,1\% del total), con una tasa de 9,5 casos por cada 100.000 habitantes (59). Se ha determinado el potencial cancerígeno de las aflatoxinas encontrando que pueden jugar un papel causal entre el 4,6$28,2 \%$ de todos los casos globales de $\mathrm{CHC}(60)$, además, otros factores igualmente asociados como la cirrosis, tienen una participación entre el 60 - 90\% de casos de CHC, la diabetes del $36 \%$, el virus de la hepatitis B (VHB) entre el $10-25 \%$ y alcoholismo entre el 2,4-7\% (61). La estimación anual de la incidencia de CHC en países latinoamericanos como Argentina, Brasil y México para casos de VHB positivos, estuvo entre 6-15 casos por 100.000 habitantes, contrastado con países africanos que presentaron una incidencia entre 3-54 casos por 100.000 habitantes (60). 
La mayoría de los casos de CHC ocurren en África subsahariana, el sudeste de Asia y China, donde hay mayor prevalencia de VHB y la exposición a aflatoxinas es alta debido al consumo de cereales y frutos secos contaminados (niveles entre 18-480 $\mu \mathrm{g} / \mathrm{kg}$ en maíz en Kenia, $0,18-36,1 \mu \mathrm{g} / \mathrm{kg}$ en cereales para desayuno en Ghana, $13,5 \mu \mathrm{g} / \mathrm{kg}$ en maíz y $27,53 \mu \mathrm{g} / \mathrm{kg}$ en maní en China $(28,32,62))$. Se ha confirmado que AFB1, por acción de la función microsomal hepática, forma el compuesto AFB1exo-8,9epoxido, el cual es altamente inestable y se une con alta afinidad a la guanina. Este aducto causa errores en las subsecuentes transcripciones del ADN, causando tumores en ratas y también en el hígado de los seres humanos (63).

\section{Evaluación de la exposición}

Para determinar la ingesta de sustancias contaminantes como las aflatoxinas es necesario estimar la exposición dietaria, que se obtiene mediante la información disponible sobre la cantidad de alimentos contaminados consumidos, la concentración de sustancias químicas detectadas y el peso corporal promedio para el grupo de personas en el que se va a realizar la evaluación (64). Uno de los métodos para estimar la exposición a contaminantes es el margen de exposición (MOE por sus siglas en inglés), que proporciona una indicación del nivel de peligro sanitario sobre la presencia de una sustancia en los alimentos sin cuantificar el riesgo. El MOE tiene en cuenta la potencia relativa de cáncer y estimaciones de la exposición, y por tanto da una indicación del nivel de preocupación (65) y relaciona la ingesta total y una referencia toxicológica (generalmente el límite inferior de un nivel de dosis de referencia que causó $10 \%$ de incidencia de cáncer en roedores). Cuanto más elevado es el MOE, menor es la preocupación sobre la salud. Si se dispone de datos adecuados para determinar que el mecanismo de acción del poder cancerígeno es genotóxico, EFSA ha sugerido que sería conveniente un MOE de 10.000 o más, es decir, un valor inferior a 10.000 puede indicar un problema de salud humana (66).
En 2001 se investigaron las micotoxinas más importantes (incluyendo aflatoxinas) presentes en la dieta de la población adulta e infantil en Suecia (67), comparando la ingesta diaria tolerable para cada toxina analizada. Aunque en la mayoría de alimentos analizados los niveles de las micotoxinas evaluadas no superaron los límites máximos permitidos según la Unión Europea $(2 \mu \mathrm{g} / \mathrm{kg})$, en alimentos como las nueces del Brasil y pistachos se hallaron altas concentraciones de aflatoxinas $(>10 \mu \mathrm{g} / \mathrm{kg})$, resultando en una elevada ingesta para las personas que consumen este tipo de productos y permitiendo concluir que, a pesar de que la exposición a las demás micotoxinas analizadas (ocratoxina A, patulina y tricoticenos) no representaban mayor riesgo para la salud, las altas concentraciones de aflatoxinas enfatizaron la necesidad de prevenir y generar mecanismos de control.

En 2004 se reportó en Francia la estimación de la exposición a aflatoxinas con el estudio de la dieta total francesa y se comparó con la ingesta diaria tolerable para varias micotoxinas (aflatoxinas, ocratoxina A, tricoticenos, zearalenona, fumonisinas y patulina) (68). A pesar de que los resultados mostraron que los niveles de contaminantes cumplían con la legislación de ese país, se concluyó que es necesario prestar especial atención a la exposición de grupos particulares como la población infantil y los vegetarianos, quienes podrían estar más expuestos a contaminación en concentraciones que excederían los límites tolerables o los niveles de ingesta semanal de estas micotoxinas.

Durante 2004 en Kenia, se identificaron 317 casos de insuficiencia hepática aguda, de los cuales 125 resultaron en muertes (69). Estos fueron atribuidos al consumo de maíz contaminado por aflatoxinas, pues se encontraron concentraciones de AFB1 hasta 220 veces superiores al límite máximo sugerido por las autoridades de ese país (20 ppb). Debido a la alta prevalencia de casos de aflatoxicosis documentados en el país, en el 2013 se cuantificó la exposición de aflatoxinas a 
través de un estudio serológico y epidemiológico transversal. Se detectaron aductos de AFB1 en el $78 \%$ de las muestras de suero y se concluyó que la exposición a aflatoxinas es un problema de salud pública en Kenia, el cual puede generar impacto en la salud humana (70).

Se ha señalado que la relación de esta toxina con el CHC se debe a la alta y frecuente ingesta de alimentos contaminados, independiente de la concentración de AFB1 que contenga cada producto, según lo reveló un estudio realizado en el 2008 la FAO/OMS (JECFA) en África (71), asegurando que, aunque se cumplan con los límites establecidos, no se garantiza la seguridad alimentaria. No obstante, para poblaciones de alto riesgo como personas con $\mathrm{VHB}$, el control de niveles máximos de AFB1 en alimentos podría protegerlos y reducir el riesgo de desarrollar CHC (72). Pese a ello, otro estudio realizado en la población adulta de Malasia determinó que la exposición a AFB1 por ingesta de alimentos listos para el consumo contribuye entre el 12,4\%$17,3 \%$ de los casos de cáncer de hígado y que la reducción de los niveles de 15 a $5 \mu \mathrm{g} / \mathrm{kg}$ es capaz de reducir hasta el 5,1\% de estos casos atribuidos a AFB1 en la dieta (73).

Por otra parte, a pesar de que en Japón se encontraron niveles que llegaban hasta los 28 $\mu \mathrm{g} / \mathrm{kg}$ en frutos secos, los resultados acerca de la ingesta de AFB1 indicaron que para 2010, el consumo de esta aflatoxina en ese país no tuvo un efecto apreciable sobre la salud (18).

Según el documento de debate sobre las aflatoxinas en los cereales, publicado por la comisión del Codex Alimentarius en 2013 (19), se estimó la exposición a estas micotoxinas en diversos países para diferentes productos teniendo en cuenta el total del límite medio superior de contaminación en estos alimentos, los grupos de consumo según la OMS y un peso corporal promedio de $60 \mathrm{~kg}$. A partir de esto, en Colombia el Instituto Nacional de Salud realizó la evaluación del riesgo de AFB1 en diferentes productos, determinando sus valores de exposición dietaria según concentraciones obtenidas en estudios realizados en el país. Los valores de exposición estuvieron entre 0,0038 - 0,182 $\mu \mathrm{g} / \mathrm{kg} / \mathrm{pc} /$ día para maíz, 0,00231 $\mu \mathrm{g} /$ $\mathrm{kg} / \mathrm{pc} /$ día para arepas y 0,01131 $\mu \mathrm{g} / \mathrm{kg} / \mathrm{pc} /$ día para alimentos para lactantes y niños, lo que contrasta con el valor de referencia tomado para el análisis que fue de $0,001 \mu \mathrm{g} / \mathrm{kg} / \mathrm{pc} /$ día (64); los resultados obtenidos demostraron que existe alto riesgo por la ingesta de esta toxina debido al alto consumo de estos productos. Para otros países latinoamericanos, la FAO realizó la evaluación de la ingesta de 13 grupos de alimentos y halló que Argentina, Chile, Venezuela y Ecuador tienen un consumo medio de cereales como el arroz y el maíz con alta contaminación por aflatoxinas; se obtuvo un MOE inferior a 10, lo que indica un posible problema de salud pública (19).

Brasil, al ser un país productor y consumidor de frutos secos como nueces, maní y almendras, ha llevado a cabo estudios donde se prioriza el análisis del riesgo por consumo de aflatoxinas. Andrade y de Mello (74) encontraron que los niveles de concentración de aflatoxinas en este tipo de alimentos se encuentra entre $6,7 \mu \mathrm{g} / \mathrm{kg}$ y $36,9 \mu \mathrm{g} / \mathrm{kg}$ y los MOE alcanzaron un rango entre 25 y 6 para la población total y para altos consumidores, respectivamente, indicando un riesgo potencial de padecer $\mathrm{CHC}$.

De la misma manera, la ocurrencia mundial de aflatoxinas B1, B2, G1 y G2 en maíz, arroz, sorgo $\mathrm{y}$ trigo fue recientemente evaluada por Andrade y Caldas. Los datos de consumo de alimentos fueron tomados a partir de 17 grupos de dietas, realizando la caracterización del riesgo derivado de la exposición a aflatoxinas a través del MOE. Dentro de los resultados encontrados se halló que la media del límite superior de las muestras contaminadas fue $13,6 \mu \mathrm{g} / \mathrm{kg}$, siendo mayor para arroz $(24,6 \mu \mathrm{g} / \mathrm{kg})$ y sorgo $(25,9 \mu \mathrm{g} / \mathrm{kg})$. En promedio, el consumo de arroz contribuyó al $41,6 \%$ de la ingesta total de aflatoxinas en todos los grupos evaluados, seguido del trigo $(35,4 \%)$, el maíz $(21,2 \%)$ y el sorgo $(1,8 \%)$. El resultado 
obtenido según el MOE (rango entre 56 y 10) indicó un riesgo potencial para los consumidores (75).

Teniendo en cuenta que, según la Comisión de la Unión Europea, el límite para la ingesta tolerable diaria de aflatoxinas en maíz y derivados es de 0,001 $\mu \mathrm{g} / \mathrm{kg}$ pc/día, los valores obtenidos según algunos de los estudios realizados estarían por encima de este referente, indicando un posible riesgo para los consumidores (Tabla 1 ). Por lo tanto, sería necesario realizar una caracterización del riesgo en cada país, con datos de vigilancia y control que hayan sido recolectados bajo muestreos y análisis validados, además, evaluar los factores asociados a la susceptibilidad de la contaminación de los alimentos más consumidos y que presentan mayor ocurrencia de aflatoxinas, la probabilidad de exposición al alimento y la probabilidad de que el alimento contenga la dosis tóxica (64).

Tabla 1. Exposición dietaria de aflatoxinas en diversos países.

\begin{tabular}{|c|c|c|c|c|}
\hline País & Alimento & Aflatoxina & $\begin{array}{c}\text { Exposición dietaria } \\
(\mu g / \mathrm{Kg} p c / \text { día })\end{array}$ & Referencia \\
\hline China & Especias & B1 & $2,8 \mathrm{E}-06$ & (3) \\
\hline Corea & Arroz & B1 & $0,0012-0,0058$ & (17) \\
\hline Japón & $\begin{array}{c}\text { Frutos secos, } \\
\text { especias, chocolate, } \\
\text { maíz }\end{array}$ & B1 & $3 E-06-4 E-06$ & (18) \\
\hline Colombia & $\begin{array}{c}\text { Maíz } \\
\text { Arroz }\end{array}$ & $\begin{array}{l}\text { B1 } \\
\text { B1 }\end{array}$ & $\begin{array}{l}0,0699 \\
0,0055\end{array}$ & (64) \\
\hline Argentina, Chile & $\begin{array}{l}\text { Maíz } \\
\text { Arroz }\end{array}$ & Totales & $\begin{array}{l}0,0064 \\
0,0011\end{array}$ & (19) \\
\hline Venezuela, Ecuador & $\begin{array}{l}\text { Maíz } \\
\text { Arroz }\end{array}$ & Totales & $\begin{array}{l}0,0047 \\
0,0075\end{array}$ & (19) \\
\hline Brasil & Maní, nueces & Totales & $0,0068-0,0276$ & (74) \\
\hline
\end{tabular}

\section{CONCLUSIÓN}

Se ha estimado que en los países en vía de desarrollo hay mayor prevalencia y exposición a aflatoxinas, ya que las regulaciones durante cosecha y poscosecha no existen o se llevan a cabo de manera inadecuada implicando deficientes prácticas agrícolas, almacenamiento inadecuado y condiciones de transporte poco óptimas; además, por las dificultades de acceso a otros productos, las dietas de muchos de estos países están basadas principalmente en alimentos susceptibles a contaminación por micotoxinas como los cereales; también las condiciones climáticas tropicales y subtropicales de algunos de ellos benefician el crecimiento de hongos y la producción de aflatoxinas (76).

A pesar de que en algunas regiones se han implementado normativas con niveles máximos permitidos, los controles de calidad agrícola y productiva son pocos o nulos (77). Se requieren estrategias de control y vigilancia más integrales para generar un mayor cumplimiento en seguridad alimentaria y asegurar que los alimentos consumidos tengan el menor riesgo posible 


\section{REFERENCIAS}

1. Harrison P. Agricultura mundial: hacia los años 2015/2030. Dirección de Información de la FAO. 2002.

2. Cano-Sancho G, Ramos AJ, Marín S, Sanchis V. Presence and co-occurrence of aflatoxins, deoxynivalenol, fumonisins and zearalenone in gluten-free and ethnic foods. Food Control. 2012; 26(2):282-6.

3. Zhao X, Schaffner DW, Yue T. Quantification of aflatoxin risk associated with Chinese spices: point and probability risk assessments for aflatoxin B 1. Food control. 2013; 33(2):366-77.

4. Wogan GN, Kensler TW, Groopman JD. Present and future directions of translational research on aflatoxin and hepatocellular carcinoma. A review. Food additives \& contaminants: part A. 2012; 29(2):249-57.

5. Ossa DEH, Hincapié DA, Peñuela GA. Determination of aflatoxin $M 1$ in ice cream samples using immunoaffinity columns and ultra-high performance liquid chromatography coupled to tandem mass spectrometry. Food Control. 2015; 56:34-40.

6. Majeed S, Iqbal M, Asi MR, Iqbal SZ. Aflatoxins and ochratoxin A contamination in rice, corn and corn products from Punjab, Pakistan. Journal of Cereal Science. 2013; 58(3):446-50.

7. IARC. IARC Monographs on the Evaluation of Carcinogenic Risks to Humans. IARC Monographs 2012; 100.

8. Wang J-S, Groopman JD. DNA damage by mycotoxins. Mutation Research/Fundamental and Molecular Mechanisms of Mutagenesis. 1999; 424(1):167-81.

9. FAO. Seguimiento del mercado del arroz de la FAO. Organización de las Naciones Unidas para la Alimentación y la Agricultura FAO. 2015; XVIII(4).

10. Diaz G, Perilla N, Rojas Y. Occurrence of aflatoxins in selected Colombian foods. Mycotoxin research. $2001 ; 17(1): 15-20$.

11. Nguyen MT, Tozlovanu M, Tran TL, Pfohl-Leszkowicz A. Occurrence of aflatoxin B1, citrinin and ochratoxin $A$ in rice in five provinces of the central region of Vietnam. Food chemistry. 2007; 105(1):427.

12. Reiter EV, Vouk F, Böhm J, Razzazi-Fazeli E. Aflatoxins in rice-a limited survey of products marketed in Austria. Food Control. 2010; 21(7):988-91.

13. Lai X, Liu R, Ruan C, Zhang H, Liu C. Occurrence of aflatoxins and ochratoxin A in rice samples from six provinces in China. Food Control. 2015; 50:401-4.

14. Commission E. Commission Regulation (EC) No. 1881/2006 of 19 December 2006 setting maximum levels for certain contaminants in foodstuffs. Off J Eur Union. 2006; 364:5-24.

15. Lutfullah G, Hussain A. Studies on contamination level of aflatoxins in some cereals and beans of Pakistan. Food Control. 2012; 23(1):32-6.

16. Aflatoxins I. IARC monographs on the evaluation of carcinogenic risks to humans. Lyon: International Agency for Research on Cancer. 1993:245-395.

17. Park J, Kim E, Kim Y. Estimation of the daily exposure of Koreans to aflatoxin B1 through food consumption. Food additives and contaminants. 2004; 21(1):70-5.

18. Sugita-Konishi $Y$, Sato T, Saito $S$, Nakajima M, Tabata $S$, Tanaka T, et al. Exposure to aflatoxins in Japan: risk assessment for aflatoxin B1. Food Additives and Contaminants. 2010; 27(3):365-72.

19. Las Aflatoxinas DdDSIC. Programa Conjunto FAO/OMS Sobre Normas Alimentarias Comité del Codex sobre Contaminantes de los Alimentos Séptima reunión Moscú, Federación Rusa, 8-12 de abril de 2013. 2013.

20. Hussain A, Luttfullah G. Reduction of Aflatoxin-B-1 and Ochratoxin-A levels in Polished Basmati Rice (Oryza sativa Linn.) by Different Cooking Methods. Journal of the Chemical Society of Pakistan. 2009; 31(6):911-5. 
21. Paliwal RL, Granados G, Lafitte HR, Violic AD, Marathée J-P. El maíz en los trópicos: Mejoramiento y producción: Food \& Agriculture Org.; 2001.

22. Karami-Osboo R, Mirabolfathy M, Kamran R, Shetab-Boushehri M, Sarkari S. Aflatoxin B1 in maize harvested over 3 years in Iran. Food Control. 2012; 23(1):271-4.

23. Rojas $V$, Martin $M$, Quinzada M. Aflatoxinas en maíz recién cosechado en Panamá. Rev méd Panamá. $2000 ; 25: 4-7$.

24. NOM NOM. 188-SSAI-2002. Control de aflatoxinas en cereales para consumo humano y animal. 2002.

25. Martínez R, Melgarejo J, García G. Inspección de aflatoxinas en maíz cultivado, almacenado y transportado en Tamaulipas, México, en 1998. Anales del Instituto de Biología serie Botánica. 74(002).

26. Speijers GJA, Speijers MHM. Combined toxic effects of mycotoxins. Toxicology letters. 2004; 153(1):918.

27. Villa P, Markaki P. Aflatoxin B 1 and ochratoxin A in breakfast cereals from Athens market: occurrence and risk assessment. Food Control. 2009; 20(5):455-61.

28. Blankson G, Mill-Robertson F. Aflatoxin contamination and exposure in processed cereal-based complementary foods for infants and young children in greater Accra, Ghana. Food Control. 2016; 64:212-7.

29. Agency FS. Survey of baby foods for mycotoxins. Food Survey Information Sheet No 68/04. 2004.

30. Ibáñez-Vea M, Martínez R, González-Peñas E, Lizarraga E, de Cerain AL. Co-occurrence of aflatoxins, ochratoxin $A$ and zearalenone in breakfast cereals from spanish market. Food Control. 2011; 22(12):1949-55.

31. Sun G, Wang S, Hu X, Su J, Zhang $Y$, Xie $Y$, et al. Co-contamination of aflatoxin B1 and fumonisin $B 1$ in food and human dietary exposure in three areas of China. Food additives and contaminants. $2011 ; 28(4): 461-70$.

32. Kilonzo RM, Imungi JK, Muiru WM, Lamuka PO, Njage PMK. Household dietary exposure to aflatoxins from maize and maize products in Kenya. Food Additives \& Contaminants: Part A. 2014; 31(12):205562.

33. Aguilera-Luiz M, Plaza-Bolaños P, Romero-González R, Vidal JM, Frenich AG. Comparison of the efficiency of different extraction methods for the simultaneous determination of mycotoxins and pesticides in milk samples by ultra high-performance liquid chromatography-tandem mass spectrometry. Analytical and bioanalytical chemistry. 2011; 399(8):2863-75.

34. Pérez JGR, Vega S, Díaz G, Urbán G, Coronado M, Escobar A. Ocurrencia de aflatoxina M1 en leches cruda, ultrapasteurizada y orgánica, producidas y comercializadas en el altiplano mexicano. Revista de Salud Animal. 2008; 30(2):53-60

35. Hernández JC, Abadía B, Moreno JdJ. Determinación de aflatoxina M1 en leche proveniente del Caribe colombiano, empleando cromatografía líquida de ultra resolución. Revista Colombiana de Ciencias Pecuarias. 2009; 22(3).

36. Signorini ML GM, Molineri A., Chiericatti CA, Zapata de Basílico ML, Basílico JC, Pisani M. Exposure assessment of mycotoxins in cow's milk in Argentina. Food and Chemical Toxicology. 2012; 50:250-7.

37. Veronezi Silva M JV, Bando E, Machinski M. Occurrence and estimative of aflatoxin M1 intake in UHT cow milk in Paraná a State, Brazil. Food Control. 2015; 53:222-5.

38. Rama A, Montesissa C, Lucatello L, Galina G, Benetti C, Bajraktari D. A study on the occurrence of aflatoxin M1 in milk consumed in Kosovo during 2009-2010. Food Control. 2016; 62:52-5.

39. Bilandžić N, Varenina I, Solomun B. Aflatoxin M 1 in raw milk in Croatia. Food Control. 2010; 21(9):1279-81.

40. Kaniou-Grigoriadou I, Eleftheriadou A, Mouratidou T, Katikou P. Determination of aflatoxin M 1 in ewe's milk samples and the produced curd and Feta cheese. Food control. 2005; 16(3):257-61. 
41. Elgerbi A, Aidoo K, Candlish A, Tester R. Occurrence of aflatoxin M1 in randomly selected North African milk and cheese samples. Food additives and contaminants. 2004; 21(6):592-7.

42. Chavarría G, Granados-Chinchilla F, Alfaro-Cascante M, Molina A. Detection of aflatoxin M1 in milk, cheese and sour cream samples from Costa Rica using enzyme-assisted extraction and HPLC. Food Additives \& Contaminants: Part B. 2015; 8(2):128-35.

43. Redouane-Salah S, Morgavi D, Arhab R, Messaï A, Boudra H. Presence of aflatoxin M1 in raw, reconstituted, and powdered milk samples collected in Algeria. Environmental monitoring and assessment. 2015; 187(6):1-4.

44. Shundo L, de Almeida AP, Alaburda J, Lamardo LC, Navas SA, Ruvieri V, et al. Aflatoxins and ochratoxin A in Brazilian paprika. Food Control. 2009; 20(12):1099-102.

45. Hernández-Hierro JM, Garcia-Villanova RJ, Rodríguez P, Toruño IM. Aflatoxins and ochratoxin A in red paprika for retail sale in Spain: occurrence and evaluation of a simultaneous analytical method. Journal of agricultural and food chemistry. 2008; 56(3):751-6.

46. Cho S-H, Lee C-H, Jang M-R, Son Y-W, Lee S-M, Choi I-S, et al. Aflatoxins contamination in spices and processed spice products commercialized in Korea. Food Chemistry. 2008; 107(3):1283-8.

47. Colak H, Bingol EB, Hampikyan H, Nazli B. Determination of aflatoxin contamination in red-scaled, red and black pepper by ELISA and HPLC. Journal of Food and Drug Analysis. 2006; 14(3):292-6.

48. Waheed S, Siddique N. Evaluation of dietary status with respect to trace element intake from dry fruits consumed in Pakistan: a study using instrumental neutron activation analysis. International journal of food sciences and nutrition. 2009; 60(4):333-43.

49. Iqbal SZ, Asi MR, Zuber M, Akram N, Batool N. Aflatoxins contamination in peanut and peanut products commercially available in retail markets of Punjab, Pakistan. Food control. 2013; 32(1):83-6.

50. Leong $\mathrm{Y}-\mathrm{H}$, Ismail $\mathrm{N}$, Latif $\mathrm{AA}$, Ahmad $\mathrm{R}$. Aflatoxin occurrence in nuts and commercial nutty products in Malaysia. Food Control. 2010; 21(3):334-8.

51. Chun HS, Kim HJ, Ok HE, Hwang J-B, Chung D-H. Determination of aflatoxin levels in nuts and their products consumed in South Korea. Food Chemistry. 2007; 102(1):385-91.

52. Neamatallah A, Serdar SA. Incidence of aflatoxins in commercial nuts in the holy city of Mekkah. Food Control. 2013; 29(1):121-4.

53. Council EEC. Commission regulation (EC). No. 1525/98. Official Journal of the European Communities. 1998; 201:43-6.

54. Masood M, Iqbal SZ, Asi MR, Malik N. Natural occurrence of aflatoxins in dry fruits and edible nuts. Food Control. 2015; 55:62-5.

55. Van de Perre E, Jacxsens L, Lachat C, El Tahan F, De Meulenaer B. Impact of maximum levels in European legislation on exposure of mycotoxins in dried products: Case of aflatoxin B1 and ochratoxin A in nuts and dried fruits. Food and Chemical Toxicology. 2015; 75:112-7.

56. Mupunga I, Lebelo S, Mngqawa P, Rheeder J, Katerere D. Natural occurrence of aflatoxins in peanuts and peanut butter from Bulawayo, Zimbabwe. Journal of Food Protection $® . ~ 2014 ; 77(10): 1814-8$.

57. Qian G-S, Ross RK, Yu MC, Yuan J-M, Gao Y-T, Henderson BE, et al. A follow-up study of urinary markers of aflatoxin exposure and liver cancer risk in Shanghai, People's Republic of China. Cancer Epidemiology Biomarkers \& Prevention. 1994; 3(1):3-10.

58. Soriano del Castillo JM. Micotoxinas en alimentos: Ediciones Díaz de Santos; 2007.

59. Ferlay J, Soerjomataram I, Dikshit R, Eser S, Mathers C, Rebelo M, et al. Cancer incidence and mortality worldwide: sources, methods and major patterns in GLOBOCAN 2012. International journal of cancer. 2015; 136(5):E359-E86.

60. Liu Y, Wu F. Global burden of aflatoxin-induced hepatocellular carcinoma: a risk assessment. Environmental health perspectives. 2010; 118(6):818-24. 
61. McGlynn KA, London WT. The global epidemiology of hepatocellular carcinoma: present and future. Clinics in liver disease. 2011; 15(2):223-43.

62. Ding $X$, Li P, Bai $Y$, Zhou H. Aflatoxin B 1 in post-harvest peanuts and dietary risk in China. Food Control. 2012; 23(1):143-8.

63. Pottenger LH, Andrews LS, Bachman AN, Boogaard PJ, Cadet J, Embry MR, et al. An organizational approach for the assessment of DNA adduct data in risk assessment: case studies for aflatoxin B1, tamoxifen and vinyl chloride. Critical reviews in toxicology. 2014; 44(4):348-91.

64. Salud INd. Evaluación de riesgo de carcinoma hepatocelular en poblacion colombiana por consumo en arepa de maíz contaminada con aflatoxina B1 (AFB1). In: Social MdSyP, editor. 2015.

65. Alimentarius FOCdC. Documento de debate sobre directrices para las opciones de gestión de riesgos sobre cómo abordar los resultados de nuevas metodologías de evaluación de riesgos. La Haya (Países Bajos), 2011.

66. (EFSA) EFSA. Opinion of the Scientific Committee on a request from EFSA related to a harmonized approach for risk assessment of substances which are both genotoxic and carcinogenic. EFSA Journal. 2005; 3(10):1-31.

67. Thuvander A, Möller T, Enghardt Barbieri H, Jansson A, Salomonsson A-C, Olsen M. Dietary intake of some important mycotoxins by the Swedish population. Food Additives \& Contaminants. 2001; 18(8):696-706.

68. Leblanc J-C, Tard A, Volatier J-L, Verger P. Estimated dietary exposure to principal food mycotoxins from The First French Total Diet Study. Food Additives and Contaminants. 2005; 22(7):652-72.

69. Azziz-Baumgartner E, Lindblade K, Gieseker K, Rogers HS, Kieszak S, Njapau H, et al. Case-control study of an acute aflatoxicosis outbreak, Kenya, 2004. Environmental Health Perspectives. 2005:177983.

70. Yard EE, Daniel JH, Lewis LS, Rybak ME, Paliakov EM, Kim AA, et al. Human aflatoxin exposure in Kenya, 2007: a cross-sectional study. Food Additives \& Contaminants: Part A. 2013; 30(7):1322-31.

71. Shephard GS. Risk assessment of aflatoxins in food in Africa. Food Additives and Contaminants. 2008; 25(10):1246-56.

72. Lee HM, Hwang JH, Ryuem TK, Jang DD, Yang J-H. Risk assessment of aflatoxin B1 from food consumption in the Korean general population. Human and Ecological Risk Assessment. 2009; 15(6):1273-85.

73. Chin C, Abdullah A, Sugita-Konishi Y. Dietary intake of aflatoxins in the adult Malaysian population-an assessment of risk. Food Additives and Contaminants: Part B. 2012; 5(4):286-94.

74. Andrade $\mathrm{P}$, de Mello MH, França J, Caldas E. Aflatoxins in food products consumed in Brazil: a preliminary dietary risk assessment. Food Additives \& Contaminants: Part A. 2013; 30(1):127-36.

75. Andrade $P$, Caldas E. Aflatoxins in cereals: worldwide occurrence and dietary risk assessment. World Mycotoxin Journal. 2015; 8(4):415-31.

76. Bhat Ramesh V, Vasanthi S. Mycotoxin food safety risk in developing countries. International Food Policy Research Institute (IFPRI), 2003.

77. Wild CP MJ, Groopman JD. Mycotoxin Control in Low- and Middle-Income Countries. Lyon, France: International Agency for Research on Cancer (IARC Working Group Report No 9). 2015. 